\title{
Corela
}

Cognition, représentation, langage

6-2 | 2008

Vol. $6, n^{\circ} 2$

\section{Anthropologie de la communication : la question du féminin en français}

Béatrice Fracchiolla

\section{OpenEdition}

\section{Journals}

Édition électronique

URL : http://journals.openedition.org/corela/286

DOI : $10.4000 /$ corela.286

ISSN : $1638-573 \mathrm{X}$

\section{Éditeur}

Cercle linguistique du Centre et de I'Ouest - CerLICO

\section{Référence électronique}

Béatrice Fracchiolla, "Anthropologie de la communication : la question du féminin en français », Corela [En ligne], 6-2 | 2008, mis en ligne le 15 décembre 2008, consulté le 01 mai 2019. URL : http:// journals.openedition.org/corela/286 ; DOI : 10.4000/corela.286

Ce document a été généré automatiquement le 1 mai 2019.

\section{(c) (i) (2)(2)}

Corela - cognition, représentation, langage est mis à disposition selon les termes de la licence Creative Commons Attribution - Pas d'Utilisation Commerciale - Partage dans les Mêmes Conditions 4.0 International. 


\title{
Anthropologie de la communication : la question du féminin en français
}

\author{
Béatrice Fracchiolla
}

\section{Introduction}

La question du genre et de la féminisation des noms de métiers est encore aujourd'hui un lieu où l'on peut observer in vivo l'évolution de la langue comme étant le reflet d'une évolution de société. Dans les années 80 , le politiquement correct - phénomène de mode ou évolution réelle du langage? - témoigne en tout cas linguistiquement d'un changement des représentations dans le «nommer l'autre». Nous pensons qu'il existe des similarités et parallélismes entre la préciosité et le politiquement correct (Fracchiolla, 2001). Or la préciosité pose également la question de la place de la femme et de son pouvoir dans la société du 17e siècle. De ce point de vue, la problématique du genre est aujourd'hui, pour l'enseignement du FLE, à la fois une problématique grammaticale et de civilisation. Enseigner une langue, c'est aussi enseigner le lien qui existe entre vocabulaire, grammaire et civilisation non seulement dans une dimension synchronique, mais aussi diachronique. La dimension synchronique permet en effet d'avoir une vision variationnelle sur une même langue (dimension francophone), alors que la dimension diachronique permet d'en avoir une explication historique. C'est pourquoi cette contribution veut tenir compte de ces deux dimensions et se situe avant tout dans le champ de la socio-linguistique. Comme le montre l'histoire de la langue et des institutions qui régissent la langue, en France, le genre s'est construit au féminin selon des structures et des lois très précises fixées à la fois par l'étymologie, l'Académie Française et la Délégation générale à la langue Française. Pourtant, dans ce domaine, c'est toujours l'usage qui fait loi avant et parfois contre toutes les instances cadrantes. Or, pour le genre, il est possible que l'usage actuel (ou plutôt, les usages actuels) soi(en)t aujourd'hui en France une conséquence de la féminisation systématique qui existe ailleurs, comme au Québec, où l'on parle des «droits de la personne", et non plus des 
«droits de l'Homme ». C'est une loi du genre. Aussi se trouve-t-on souvent, en français hexagonal, devant des incohérences et des indécisions sur ce qui est 'correct' et 'non correct', dans la mesure où l'usage est parfois en contradiction avec les règles, ellesmêmes souvent édictées par les institutions.

Bien qu'elle ne soit toujours pas considérée comme des plus pertinentes, cette évolution qui tend à la féminisation systématique, entraîne parfois des difficultés pour savoir non seulement comment l'on doit dire, mais aussi ce qu'il convient d'enseigner et comment l'enseigner. Plusieurs questions en découlent alors : pourquoi la féminisation des noms de métiers pose-t-elle encore problème? Quels sont les textes de référence ? Comment gérer la variation francophone du traitement de la féminisation des noms de métiers? Comment enseigner le genre des animés en français? Quels outils didactiques sont à notre disposition? Et comment enseigner le féminin alors qu'il n'y a pas d'accord sur le code, puisque la règle n'est pas identique à l'usage ? Si l'on dit encore 'un médecin' et non 'une médecin', on remarque cependant une certaine incohérence sémantico-cognitive qui pose la question de la désignation d'un référent féminin par une dénomination masculine. La célèbre devinette du chirurgien en est une concrète démonstration. On propose ici de réfléchir à cette problématique à partir de cette incohérence sémantico-cognitive et à partir des apports possibles d'une approche étymologique et morphologique pour enseigner le genre féminin, tout en l'associant à une démarche sociolinguistique se rapprochant de l'enseignement de la civilisation. On examinera une série d'exemples extraits d'une recherche simple, effectuée sur Internet via le moteur de recherche Google, qui reflète certaines de ces difficultés.

\section{Etat des lieux}

\section{1a. Diversité morphologique du féminin}

Le problème posé par les noms féminins animés est d'abord celui de la féminisation des noms de métiers. Cette problématique qui a fait couler beaucoup d'encre est d'autant plus mal connue aujourd'hui qu'elle s'est réalisée différemment à travers les pays de la francophonie (Houdebine, 1992 ; Dister, 2004 ; Labrosse \& Lamothe, 1992). Cela a abouti souvent à des brouillages entre usages et recommandations et a probablement contribué à faire du français une langue latine à part - dans la mesure où l'italien comme l'espagnol féminisent les substantifs de façon naturelle, en $-a$. En ce qui concerne en revanche les féminins non animés, le problème est celui de l'absence d'explication (cultivée) alors que l'étymologie et la suffixation nous donnent d'emblée certaines indications morphologiques, historiques et évolutives qui permettent au moins de comprendre les raisons d'existence de certaines formes : ainsi les substantifs suffixés en -age sont-ils du genre masculin, alors que ceux suffixés en -tion sont de genre féminin car issus de substantifs latins en -io, ionis déjà féminins (ratio, ionis > la ration; attentio, onis > l'attention; formatio, onis > la formation). Dans les deux cas, il s'agit en réalité d'un problème de repérage, pour lequel le recours à l'histoire de la langue peut nous aider. Par exemple les mots en -eur / -euse désignant des animés sont dérivés de verbes et expriment l'agentivité de celui/celle qui fait l'action (crier donne ainsi crieur, crieuse; coiffer, coiffeur, coiffeuse ; etc.). Pourtant, certains mots en -eur dérivés de radicaux de type verbal sur ce modèle sont accompagnés de formes diverses de féminins : trieur/trieuse; mais aussi éditeur/ éditrice (Huot, 2001). Parallèlement, on trouve également d'autres mots 
en -eur qui ne sont pas d'origine verbale et faisaient leur féminin en -esse: docteur/ doctoresse; vengeur/vengeresse. À ces formes s'ajoutent encore une forme comme auteur / auteur. Enfin, pour les mots en -teur / -trice, il faut regarder l'étymologie latine, la racine et le suffixe: geni - tor / gene - trix. Cet exemple d'alternance permet de mesurer la complexité créatrice liée à la féminisation nominale.

Enfin, même si certaines règles étymologiques indiquent la formation des féminins en français, certains suffixes comme celui en -esse (de doctoresse, poétesse, chasseresse...) ne sont plus productifs, et cela avant tout pour des raisons qui découlent à la fois d'une certaine désuétude mais probablement aussi d'une certaine paresse articulatoire.

\section{1b. Perspectives historiques sur la féminisation des noms de métiers}

5 L'ouvrage Femme, j'écris ton nom... Guide de féminisation de la langue française (désormais GFF, 1999) nous informe que jusqu'au 16e siècle, la langue française n'éprouvait guère de difficulté pour féminiser tous les noms de métier, y compris «nobles », qui nécessitaient de l'être parce qu'une femme les exerçait. L'on trouvait ainsi des formes comme chirurgienne et medecineuse ou médecine sans que l'homonymie soit considérée problématique (GFF : 1999, 14 et 24). Puis, aux 17e et 18e siècles, la féminisation a été totalement ignorée pour réapparaître timidement au 19e et 20e et perdurer jusqu'à nos jours, du moins pour les "petits métiers». Pourtant elle reste encore « une question d'actualité, essentiellement dans les mentalités des hommes et surtout des femmes de ce pays, puisque, à quelques exceptions près, elle est grammaticalement possible » (GFF : 1999 , 32) mais rencontre toujours certaines résistances de principe dans l'usage.

6 Par ailleurs, cette évolution a été un temps soutenue également par certaines introductions dans le dictionnaire de l'Académie française de 1932-1935 (artisane, attachée, auditrice, aviatrice, avocate, bûcheronne, candidate, compositrice, éditrice, électrice, employée, exploratrice, factrice, pharmacienne, postière, rameuse), lequel a également accordé les deux genres à un grand nombre de noms de professions impliquant des études supérieures qui n'étaient qu'au masculin dans l'édition de 1878 (archiviste, chimiste, géographe, latiniste et linguiste). Le dictionnaire de l'Académie avait également introduit chauffeuse «celle qui conduit une automobile» qui ne figure pas dans l'édition de 1992 bien que l'académicienne Marguerite Yourcenar l'emploie déjà en 1979: «Une jeune femme de village qui sert de "chauffeuse» et comme on disait autrefois dans le style noble, de " coursière ». Enfin, de nombreuses dames du village [...] apportent [...] des offres de service avec leur voiture lorsque Jeanine la chauffeuse [...] est autrement occupée ». Paul Guth en fait également usage en 1951: «les chauffeuses des délégations de Grande-Bretagne (à l'ONU) ». Pourtant, si l'homonymie a dû jouer en défaveur de ce mot que l'on ne trouve plus guère dans cet usage, personne ne songe à appeler l'ouvrier qui installe et répare les cheminées et appareils de chauffage, autrement que fumiste, ni celui qui élève des moutons que moutonnier (GFF: 1999, 32). L'Académie Française constitue pourtant aujourd'hui l'un des principaux fronts de résistance à la féminisation des noms de métiers. Elle argue d'un neutre associé à la fonction, en vertu de quoi elle prône «Madame le Ministre », etc. Pourtant, le neutre n'existe pas en français... Par ailleurs, hormis la France, les autres pays francophones semblent, comme nous le verrons, plus à l'aise, pour accepter et pratiquer la féminisation. Marguerite Yourcenar n'était-elle pas, d'ailleurs, académicienne et Belge? 


\section{1.c. La construction idéologique des genres}

7 Les premières règles d'accord de genre en français apparaissent au 17e siècle en même temps qu'une réflexion sur la langue. On voit aisément comment à partir de ces règles et en particulier de la règle selon laquelle le masculin «l'emporte » sur le féminin émerge la création culturelle d'une problématique idéologique, elle-même fondée sur des principes historiques, masculins et arbitraires que le mouvement précieux a accompagnés : « Pour une raison qui semble commune à toutes les langues que le genre masculin étant le plus noble doit prédominer toutes les fois que le masculin et le féminin sont ensemble» (Vaugelas : 1647, p. 27); ou encore : « Le plus fort l'emporte pour dire que les plus puissants ont toujours l'avantage. Masculin qualifie le plus noble des genres, celui qui a ordinairement l'avantage sur l'autre.» (Furetière: 1690, p. 29). Mais aussi : « Le genre masculin est réputé plus noble que le féminin à cause de la supériorité du mâle sur la femelle." (Beauzée: 1767). Et enfin: "Les mots masculins sont du premier genre puisqu'ils expriment la chose avec un rapport au mâle, ou comme étant de ce premier sexe. Les mots féminins sont désignés par leur appartenance au second genre ou second sexe» (Girard : 1747).

On voit ainsi comment les difficultés liées à la féminisation des noms de métiers trouvent leurs origines idéologiques et politiques dans la société du 17e siècle, pour ressurgir dans les années 80 , via le Québec, en raison de l'accession progressive des femmes à des postes à haute responsabilité, dont les titres ne se déclinent qu'au masculin. Aujourd'hui, cette problématique éminemment politique, dans la mesure où elle touche à la place des femmes dans la cité et dans la société, est devenue, pour le linguiste, une problématique sémantico-pragmatique et discursive. Il s'agit en effet de donner un nom à et de légitimer par ce nom, dans la réalité de leurs fonctions sociales, de nouveaux référents féminins, sans ambiguïté (Madame la ministre, la chirurgienne, la médecin...).

\section{La situation actuelle}

9 Nous choisissons ici de procéder de manière chronologique, en commençant par nous intéresser au Québec, premier pays francophone à avoir adopté une véritable démarche de féminisation. Nous verrons ensuite la Belgique pour arriver en dernier lieu à la politique de féminisation des noms de métier en France, qui est finalement la plus récente et en même temps la plus résistante.

\section{2a. Au Québec}

Un premier guide de féminisation linguistique est pensé en 1980 à l'université de Québec à Montréal. Son objectif simple et pragmatique est de répondre à la fois à l'accès croissant des femmes à divers postes au sein de l'université et à l'augmentation de la population étudiante féminine - passée de $45 \%$ à $60 \%$ depuis les années 70 . Il s'agit « d'harmoniser la langue avec la nouvelle réalité, rendant les femmes aussi visibles dans la langue qu'elles le sont devenues au sein de secteurs de plus en plus diversifiés de la communauté universitaire ". C'est ainsi que le conseil d'administration de l'université fait suite à la demande des professeures du Groupe Interdisciplinaire d'Enseignement et de Recherche sur les Femmes et reconnaît officiellement l'utilisation des titres féminins pour désigner 
les femmes. En 1981, un comité de féminisation est créé, qui établit une liste féminisée de tous les titres et fonctions, et établit un code syntaxique de féminisation s'appliquant aux textes de l'UQAM.

11 Au sein de l'université, l'évolution se fait en plusieurs temps. Les formulaires sont d'abord féminisés (validation en 1984), puis les règlements et les politiques (validés en 1990). Une première brochure est ensuite mise au point en 1992 pour les autres documents - tels que les textes informatifs. Dans cette brochure se trouvent explicitées les différentes stratégies possibles: "solutions alternatives» ou «stratégies de rédaction» qui permettent de témoigner de la présence des deux sexes autrement qu'en faisant coexister le nom féminin et le nom masculin. Parmi les règles et stratégies développées pour faciliter la féminisation au sein de ces documents informatifs sont proposés par exemple ( Guide de féminisation, UQAM, 1992) :

1. L'ordre masculin-féminin ou l'inverse (une étudiante ou un étudiant inscrit; un étudiant ou une étudiante inscrite)

2. L'utilisation des conjonctions « et » ou bien « ou »

3. L'accord en genre et en nombre des adjectifs et participes passés épithètes avec le nom le plus proche, et non plus systématiquement, le masculin. (Ex: de nombreuses étudiantes et étudiants/De nombreux étudiants et étudiantes / Les étudiants et étudiantes inscrites)

Dans les formulaires à remplir de l'université, on préconise «pour féminiser les noms ayant une forme différente au masculin et au féminin, _d'écrire_ au complet le terme masculin et le terme féminin, en les séparant par une virgule, sans laisser d'espace après celle-ci »: Homme,femme. La ponctuation prend alors ici un sens conventionnel tout à fait particulier, qu'il n'est guère évident d'enseigner dans un cours de langue, sauf à en généraliser l'application dans toute la francophonie. Dans les documents de type juridique (officiel), on préconise : une étudiante, un étudiant inscrit alors que l'on préfère : un-e étudiant-e inscrit-e. pour les formulaires (de type mixte).

Toutes ces possibilités figurent ici des propositions de base, tout en maintenant l'ouverture sur la possibilité d'adopter d'autres types de formulations. Parallèlement des procédés syntaxiques de féminisation sont également proposés. Il s'agit par exemple de remplacer le terme de désignation par : le nom de la fonction (responsabilités de tutorat vs tuteur); le nom de l'unité administrative (le service des archives vs un archiviste; un générique (la population étudiante vs les étudiants); un adjectif indéfini (chaque membre $v s$ chacun des membres); un pronom relatif épicène (qui vs lequel) ; un pronom personnel (leur vs à ces derniers); un élément épicène de même catégorie (les responsables vs le ou la responsable); une autre désignation (de leur part vs pour eux). Ou bien l'on peut encore: substituer la voix active à la voix passive; biffer le terme de désignation redondant; restructurer la phrase; modifier la mise en page; remplacer le terme de désignation et un autre élément de la phrase par: un nom qui leur correspond; un adjectif possessif suivi d'un nom ; une tournure impersonnelle ; un verbe à l'infinitif ; un adverbe ou une autre expression invariable...

On voit, par tous ces exemples, que les questions soulevées concernent non seulement la morphologie, mais également la syntaxe et qu'il s'agit finalement, au Québec, de contourner des caractéristiques de la langue qui apparaissent ou pourraient être perçues comme - historiquement? - sexistes. En ce sens, l'idée sous-jacente est celle de l'influence sociale que les locuteurs ont sur leur langue pour la contraindre à évoluer. On retrouve ce type d'influence dans le politiquement correct, mais en réalité, c'est en 
France au 17e siècle, que ce type de démarche prend sa source, avec le mouvement de la préciosité (Fracchiolla, 1996 ; 2002).

\section{2b. En Belgique}

15 le 21 juin 1993, suivi de l'arrêté d'application le 13 décembre. L'objectif pragmatique déclaré est de « rendre service aux fonctionnaires de l'administration de la Communauté française et aux usagers de la langue française dans leur ensemble ». De facto, ce décret a aidé l'usage des formes féminines à se développer aussi bien dans les médias - presse écrite, radio, télévision - que dans la vie courante (Dister, 2004). Cependant, deux obstacles à son application sont identifiés. Tout d'abord, dans l'administration, une enquête a révélé le poids de décision des chefs de service (on féminise selon leur choix). Le second obstacle est une forme de résistance à la féminisation chez certaines femmes, qui occupent pour la première fois un poste jusqu'alors réservé à un homme : le masculin est en effet alors associé au prestige. On préconise de respecter cette demande des femmes qui finira par disparaître d'elle-même. En 1994, afin d'éviter les «créations sauvages ", le Conseil supérieur de la langue française nomme une Commission de féminisation constituée de cinq membres (Michèle Lenoble Pinson, Marie-Louise Moreau, Marc Wilmet, linguistes et deux parlementaires). La Commission rédige alors le livret Mettre au féminin, édité en 1994, qui comportait le rappel des règles de féminisation, cinq recommandations (dont généraliser « Madame » à la place de " Mademoiselle »), ainsi que la liste alphabétique de 1500 noms féminins. Dix ans après, l'usage a évolué et des formes comme auteure et écrivaine, mises en note en 1994, se disent couramment.

Une seconde édition de Mettre au féminin (publiée par le Service de la langue française de la communauté française de Belgique) est parue en 2005 (avec une liste de 1619 entrées mise à jour par une nouvelle Commission de féminisation, composée des cinq précédents membres plus Jean-Marie Klinkenberg et Georges Legros). Par son travail de récolte de données attestant des formes féminines depuis 94 , en particulier en dépouillant la presse (Fujimura, 2005; Dister, 2004), le rôle de Michèle Lenoble-Pinson s'est révélé particulièrement essentiel dans la constitution de cette liste.

En Belgique comme au Québec l'accent est mis sur la liberté des utilisateurs. Ainsi préconise-t-on ici la coexistence (une professeur // une professeure); (la maire // la mairesse), etc. "Là où les normes n'apparaissent pas encore clairement, ce sont les pratiques des usagers qui construiront peu à peu " (Mettre au féminin, 2005). Les règles de 2005 sont assez semblables à celles de la circulaire française de 86 (jamais appliquée); les usages rejoignent ceux préconisés déjà officiellement en Belgique en 1994 et, à titre indicatif, en France depuis 1999 (une chercheuse, une professeur, une auteur). Mais "Depuis lors, on peut constater que les féminins en -eure s'implantent dans les habitudes d'usagers de plus en plus nombreux, en Belgique, en France, au Québec et en Suisse romande. Dans ces cas, le Conseil ouvre à présent totalement le choix entre les formes classiques en -eur et celles en -eure, qui assurent davantage la visibilité des femmes, en particulier lorsque les mots sont accompagnés de déterminants élidés ou au pluriel (l'auteure, les auteures vs l'auteur) » (Mettre au féminin, 2005). 


\section{2c. En France}

En 1984, Yvette Roudy, alors ministre des droits de la femme, met en place avec Benoîte Groult une commission de terminologie chargée de la féminisation des noms de métiers et de fonction, qui aboutit en 1986 à la Circulaire du 18 mars relative à la féminisation des noms de métiers - fonctions, grade ou titre parue au Journal officiel de la République Française, $p .4267$. Pourtant, cette circulaire très utile et qui établit certaines règles, n'a jamais été appliquée. En revanche, elle a servi de base à Lionel Jospin qui, Premier ministre, a mis en place à son tour une deuxième commission générale de terminologie et de néologie (Rapport d'octobre 1998). Ce travail aboutit au guide intitulé Femme, j'écris ton nom...Guide d'aide à la féminisation des noms de métiers, publié en 1999 (GFF), et présentant une liste à caractère exhaustif. Cependant, quoique très utile, aucun texte officiel ne contraint ni l'administration, ni l'usager, à appliquer les recommandations de ce texte. Les caractéristiques de l'ouvrage sont d'être très ouvert, en particulier sur les solutions francophones déjà répandues, et de s'en remettre globalement à la sanction évolutive de l'usage. En particulier, le texte reprend à son compte toutes les formes existantes de la francophonie (Belgique, Québec, Suisse). Un problème majeur subsiste cependant: n'ayant aucune inscription légale, ce guide reste, dix ans plus tard, toujours méconnu du grand public, et l'absence de position officielle de la France contribue à brouiller les esprits. Aussi, ce sont toujours aujourd'hui les mêmes questions qui sont posées de manière récurrente, comme l'atteste l'exemple ci-dessous :

"Je suis prof de français dans une universite americaine, et auteur(e) d'un manuel de francais pour debutants. Je voudrais mettre dans le manuel le feminin "la professeure", mais j'ai du mal a savoir a quel point cette forme s'emploie dans la langue de tous les jours en France, bien que recommandee par les commissions officielles, meme en France. Je sais que les Francais (et Francaises) ont ete plus lents a adapter ces nouveaux usages, mais est-ce que les gens ne commencent pas a changer d'habitude? Je sais que 'la prof' est tres repandu en francais familier. Ou en est-il de 'la professeur' ou 'la professeure' dans les usages un peu plus formels (oraux, aussi bien qu'ecrits) ? Il y a des etudes linguistiques qui ont ete faites sur la presse, mais je m'interesse aux usages des gens ordinaires. Merci d'avance!» (Posté le : Mar Sep 26, 20068 :01 pm Sujet du message : La professeure ?)

http://www.francparler.info/forum/viewtopic.php?t=1406

Or, comme nous l'avons dit précédemment, cette problématique, parce qu'elle renvoie aussi à la place des femmes dans la cité et dans la société, est devenue pour le linguiste une problématique sémantico-pragmatique et discursive. On constate en français deux formes d'inégalité entre les genres dans la langue: d'une part une dissymétrie grammaticale (l'accord de principe au masculin) dont nous avons vu les origines et qui continue à être la règle générale - sauf à quelques rares exceptions comme celle de l'UQAM à Québec, et d'autre part une dissymétrie sémantique que nous allons tenter d'expliquer ci-après.

\section{2d. Dyssymétrie grammaticale}

Afin de pallier les dissymétries grammaticales, le québecois, en particulier, favorise certaines créations qui s'exportent plus ou moins en France au vu des utilisations statistiques de l'usage. Les créations de féminins obéissent aujourd'hui à certaines règles qu'il est possible de résumer comme il suit. À partir du modèle général d'alternance dans 
la finale des mots, on trouve ainsi formés, à partir de travailleur / travailleuse, les termes ingénieuse (qui peut être nom ou adjectif) ou procureuse ; et auteur / autrice créé à partir de compositeur / compositrice. De la même manière, sur l'alternance -er / -ère ont été créés policière et menuisière alors que le modèle -ien / -ienne a donné chirurgienne et écrivaine. Moins faste, le modèle de courtisane a donné partisane ou encore artisane ; alors qu'avocate a produit soldate, clocharde. Et avec un succès très variable, bédouin/ bédouine a produit témouine...

\section{2e. Dissymétries sémantiques}

D'un autre type, les dissymétries sémantiques touchent des mots tellement courants qu'on y prête rarement attention. Elles proviennent de l'inégalité de sens entre un mot masculin et son féminin. On remarque que, souvent, et sans réelle explication autre qu'une épaisseur de sens (Robert, 2000) déposés au fur et à mesure des usages et d'une dépréciation systématique du féminin comme "(sexe) faible » et opprimable, le mot féminin comporte une nuance péjorative, négative ou carrément dégradante alors que le masculin se veut neutre ou noble. Pour illustration, voici quelques exemples :

\begin{tabular}{|l|l|}
\hline Homme galant (bonnes manières) & Femme galante (prostituée) \\
\hline Garçon (neutre) & Fille (fille de joie) \\
\hline Gars (neutre) & Garce (négatif, péjoratif) \\
\hline Courtisan & Courtisane (prostituée) \\
\hline Gouverneur (pouvoir) & Gouvernante (service) \\
\hline Maître (pouvoir) & Maîtresse (sexuel) \\
\hline Patron (pouvoir) & Matrone (tenancière d'auberge, désuet) \\
\hline Compère (ami) & Commère (bavarde) \\
\hline Entraîneur (sportif) & Entraîneuse (service sexuel) \\
\hline Allumeur (professionnel) & Allumeuse (femme séductrice/négatif) \\
\hline Homme facile (à vivre) & femme facile (sexuel, péjoratif) \\
\hline
\end{tabular}

\section{Usages et méthodes}

\section{3a. L'influence québécoise de la féminisation en -e (chercheure, professeure, auteure). Aperçu de l'usage écrit électronique}

22 Afin d'obtenir un instantané rapide des usages réels, nous avons effectué une recherche sur Internet, via Google, en date du 15 mars 2008, qui portait sur trois requêtes de 
féminisations caractéristiques : chirurgienne, auteure, autrice, chercheur(e) et chercheuse. Les résultats obtenus sont les suivants :

La première requête lancée sur femme chercheur en français, trouve sans difficulté sur des sites français de nombreuses expressions soit au singulier, soit au pluriel: «femme chercheur ", "chercheur femme ", où chercheur - forme masculine - est mis en épithète à femme, constituant presqu'un mot composé : «Place et nombre de chercheurs femmes dans la recherche; la part des femmes parmi les chercheurs a doublé; le poids des femmes chercheurs travaillant dans...; 1652 chercheur-e-s dont 3634 femmes; Elle est femme chercheur de l'Institut de Physique des Hautes Energies de l'Académie des Sciences de Chine». La requête sur la forme chercheuse, qui serait, d'après la règle, la forme morphologique correcte de féminisation issue du verbe "chercher", donne d'abord comme premier résultat "la chercheuse», ici le titre d'un site annuaire - donc, ne renvoyant absolument pas à un animé féminin comme attendu. On trouve ensuite en effet, toujours sur des sites français, des occurrences de féminisation du nom de métier : "Après des activités de chercheuse entièrement centrées sur la physique ", "une chercheuse et documentaliste de film à la double nationalité française et iranienne », " Chercheuse au département "Écologie et modèles pour l'halieutique » du Centre Ifremer de Nantes ». La requête sur chercheure aboutit en revanche d'abord systématiquement sur des sites québécois, tout en donnant de nombreuses occurrences: "Portrait d'une chercheure "; " Prix jeune chercheure »; « une chercheure de l'université Laval reçoit près de 365000 \$", « chercheure basée à Georgetown ». On trouve cependant aussi, mais beaucoup plus rarement (deux occurrences sur dix résultats, en première page de requête Google), l'expression sur des sites français (CNRS d'Ivry ou Inserm) : «Chercheure hors-statut mais ethnologue »; "Enseignant-chercheure déléguée au CNRS du 1er septembre 2003 », " Chercheure à l'INSERM... ».

Ces exemples montrent combien l'usage lui-même est flou en langue française, laquelle est indifférenciée sur Internet dans la mesure où l'ancrage national des sites est souvent peu évident au premier abord. S'il y a en effet une règle de formation morphologique des féminins de noms de métiers dérivés de verbes en français hexagonal, ce n'est pas celle qui a été choisie au Québec, où on ajoute simplement un - $e$ en finale. Or, nous postulons que le québécois semble contaminer le français via certains milieux (universitaires, en particulier, comme l'attestent la plupart des exemples) qui, plus éduqués et cultivés, sont encore plus à même de réagir à l'absence de règle volontariste claire et définie en français. On voit également comment il peut y avoir une contamination seconde par exemple de professeure à chercheure (plutôt que chercheuse) en français : le mot professeur n'étant pas constitué à partir du verbe professer, mais du substantif latin professio, onis, il connaît, lui, une féminisation en -e. Les résultats obtenus sur la requête " professeure " sont en tout cas, ici, d'abord français et québécois ensuite, dans une proportion inverse à celle de chercheure, soit huit résultats pour les sites français (dont «la professeure poignardée ») contre deux résultats pour le Québec.

Auteure ou autrice? Là encore, le français recommande une formation en -trice, alors que le québécois réalise une féminisation simple en - - . Les résultats obtenus sur autrice sont très variés et vont du québécois à l'italien (où la forme est régulière) en passant par le français de façon incertaine (toujours en raison d'une identification nationale linguistique des sites parfois difficile). On trouve au passage dans un blogue une utilisation justifiée de manière tout à fait intéressante. Si l'on en croit "l'autrice » précisément, la forme a été utilisée dans le Mercure de France, dans les années 30. Pour 
auteure, en revanche, Wikipédia renvoie directement à un usage suisse et québécois, mais la première page de requête montre des usages personnels français nombreux, en particulier et au moins sur trois blogues personnels de femmes, alors que les usages québécois du terme se trouvent sur des sites plus officiels, de 'vraies' auteures. On peut déduire de ces exemples une tendance sociolinguistique évolutive naturelle, qui tend à la simplification par uniformisation du modèle comme par homogénéisation d'une règle normalement adjectivale en français, qui consiste à former les féminins par l'ajout simple d'un $-e$, reconnue par les diverses variétés du français comme forme morphologique essentielle du féminin. Enfin, pour chirurgienne, l'usage statistique est très clair: les usages sur des sites français sont uniquement de type personnel individuel (blogues, forums), alors que le terme est utilisé sur des sites institutionnels québécois, révélant par là un écart intéressant entre les politiques linguistiques.

\section{Conclusion} «-oi» fut persistante jusqu'en 1848 pour «-ai», alors que tout le monde opérait depuis longtemps une distinction sonore. Plus proche de nous, pensons au passage progressif de "événement " à "évènement ", finalement consigné lui aussi, à égalité, dans le dictionnaire; ou encore, enfin, à la disparition de ces accents circonflexes après que la majorité des locuteurs a renoncé à leur usage phonologique distinctif (" pâtes »/ «pattes »). Or, quelle que soit l'origine des tentatives de cadrage formel de la langue, des Académiciens, ministres, ou autres autorités linguistiques, le développement d'une langue est avant tout oral (voire gestuel!), et l'usage oral demeure premier par rapport à l'écrit.

« (...) En français, comme le genre est soutenu dans de nombreux cas par la mémoire des locuteurs natifs pour chaque unité lexicale, on n'a aucun moyen de prévoir, de façon générale, le genre de personne sans connaitre la particularité de ce mot » (Fujimura, 2005 : 50). Cet argument justifie alors d'autant plus une féminisation référentielle. Il s'agit de défendre l'usage, et un usage pragmatique de la langue, où l'on prend en compte le sens via le référent et non via l'étymologie, dans le dessein de mener une politique linguistique positive de la francophonie. La problématique rappelle aussi dans une certaine mesure celle de la Défense et illustration de la langue française de Du Bellay (1549) et les problèmes de retranscription et d'unification graphique et sonore qui se posèrent alors. Pourquoi, aujourd'hui, cette évolution pragmatique de la langue, n'est-elle pas de fait une règle généralisée ? et pourquoi la féminisation des noms de métiers devrait-elle être traitée sur un autre plan que celui-là ? Comment enseigner la règle, quand c'est l'usage qui est la règle et que l'usage est multiple? Cependant, en partie grâce au développement d'Internet et des technologies de l'information et de la communication, qui créent une véritable perméabilité des usages et vont dans le sens d'une globalisation linguistique, on voit que l'usage converge dans le sens de règles francophones communes d'acceptation et de tolérance, et ce, malgré les résistances. En matière d'enseignement (qui forme les futurs utilisateurs/locuteurs de la langue, et donc les futures pratiques de celle-ci), nous ne pensons pas qu'il soit nécessaire d'éviter alors un traitement exhaustif des formes, y compris dans les méthodes qui s'adressent à des débutants, au contraire. Développer de façon précoce dans l'apprentissage l'existence de la problématique exceptive par rapport à une règle (qui est donc multiple et tient compte des phénomènes de variations) rend 
bien compte d'une dynamique de la langue qui est, également, une dynamique de civilisation. Le traitement du genre est ici plutôt amputatif d'une réalité, au sens où il perpétue une forme de non-dit qui contribue à brouiller les repères linguistiques mais aussi civilisationnels. Une approche ouverte serait au contraire l'occasion d'une perspective francophone sur la langue, généralement négligée, voire occultée. On reste ainsi dans un enseignement dichotomique 'figiste' du français qui ne prépare pas à une prise de contact réelle avec les terrains francophones et variationnels divers constituant le vécu de la langue. Comment réagira en effet notre apprenant grand débutant quand, arrivant en France, il sera étonné par un débat qu'il ne comprend pas, mais qui existe toujours, sur ce qui est féminisable ou pas ; ou encore arrivant au Québec ou en Suisse, il sera décontenancé par certaines formes, qu'il n'a pas apprises (une professeure) ? Aussi, développer de façon précoce chez les débutants l'existence de la problématique permettrait de rendre compte d'une dynamique de la langue qui est, également, une dynamique de civilisation. Ne pas traiter le genre des animé(e)s en revanche paraît entretenir un non-dit, et contribuer à brouiller les repères linguistiques mais aussi civilisationnels, alors que cela pourrait être ici justement l'occasion de traiter d'une ouverture francophone sur la langue, qui existe rarement. C'est ainsi qu'on reste dans un enseignement du français qui ne prépare guère à la rencontre des réalités de la langue.

\section{BIBLIOGRAPHIE}

Beauzée, Nicolas, (1767), Grammaire générale, ou Exposition raisonnée des éléments nécessaires du langage pour servir de fondement à l'étude de toutes les langues, imprimerie Babou, 2 vol. in $8^{\circ}$ (republié en 1971, chez Hachette).

Délégation à la langue française, http://www.culture.gouv.fr/culture/dglf/garde.htm Dister, Anne, (2004), « La féminisation des noms de métier, fonction, grade ou titre en Belgique francophone. Etat des lieux dans un corpus de presse », JADT 2004 : 7èmes Journées internationales d'Analyse statistique des Données Textuelles, p. 313-324.

Egalité des sexes et développement, concepts et terminologie / Agence intergouvernementale de la francophonie (nov.2002), Les noms de métiers du Ministère se disent aussi au féminin/ Brochure du Ministère des affaires sociales, du travail et de la solidarité. 2002 Circulaires et Notes de service du Ministère de l'Agriculture, de l'Alimentation, de la pêche et de la Ruralité Sommaire $n^{\circ} 20$ du 19 mai 2005.

Féminisation des noms de métiers, JOURNAL OFFICIEL DE LA REPUBLIQUE FRANCAISE, 18 mars 1986, page 4267, Circulaire du 11 mars 1986 relative à la féminisation des noms de métiers, fonction, grade ou titre.

Femme,j'écris ton nom... Guide d'aide à la féminisation des noms de métiers, titres, grades et fonctions, (1999), Centre national de la recherche scientifique, Inalf, téléchargeable sur : http:// www.ladocumentationfrancaise.fr/BRP/994001174/0000.pdf

Fracchiolla, B., (2002) : « Langue et culture : le politiquement correct », Culture pour tous, éditions Bérénice, Paris, p. 141-158. 
Fracchiolla, DEA, 1996

Furetière, A., Dictionnaire universel, 1690.

Fujimura, Itsuko, (2005) « La féminisation des noms de métiers et des titres dans la presse française (1988-2001) », Mots. Les langages du politique, $n^{\circ} 78$, juillet, p. 37-52.

Girard, Les vrais principes de la langue française,1747.

Guide de féminisation ou la représentation des femmes dans les textes, UQAM, Université du Québec à Montréal, 06/03/2008, 19 pages. http://www.instances.uqam.ca/guide/ guide_feminisation.html

Houdebine, Anne-Marie, (1992), «Sur la féminisation des noms de métiers en français contemporain », Recherches féministes, vol. 5 n 1, p. 153-159.

Huot, Hélène, Morphologie. Forme e sens des mots du français, Campus, Armand Colin, Paris, 2001.

Labrosse, Céline et Lamothe, Jacqueline, (1992), « Un fragment du féminisme québécois des années 80 : la féminisation linguistique », Recherches féministes, vol. 5 nº 1, p. 143-151.

Labrosse, Céline, (1996), Pour une grammaire non sexiste, Les éditions du remue ménage, Montréal.

Mettre au féminin. Guide de féminisation des noms de métier, fonction, grade ou titre. Service de la langue française, , 06/03/2008.

Paveau, M.-A., Rosier, L., La langue française. Passions et polémiques, Paris, Vuibert, 2008.

Robert, S., (2003), « L'épaisseur du langage et la linéarité de l'énoncé : vers un modèle énonciatif de production et d'interprétation de l'énoncé », Ouattara, Aboubakar (éd.), Parcours énonciatifs et parcours interprétatifs. Théories et applications. Actes du colloque de Tromso- 20-28 octobre 2000, (Oslo), Paris, éd. Ophrys, p. 255-274.

Vaugelas, Remarques sur la langue française, utiles à ceux qui veulent bien parler et bien écrire, 1647.

Yaguello, Les mots et les femmes. Essai d'approche sociolinguistiques de la condition féminine, Payot, Paris, 1978.

\section{RÉSUMÉS}

Après une mise en perspective historique, cet article traite des difficultés communicationnelles que peuvent poser les variations francophones de la féminisation, et en particulier les féminisations des noms de métiers. On s'attachera à illustrer la situation présente par des comparaisons entre le Québec, la Belgique et la France à partir d'un corpus Internet pour conclure sur les difficultés plus larges posées par un enseignement variationnel du français.

This contribution aims at exposing the communicational difficulties caused by the variety of French language treatment of feminization around the world, and especially of the feminization of professional functions. After an historical approach, we expose the present situation through comparisons between Quebec, Belgium and France, based on an Internet corpus and the problems it leads to regarding the teaching of French language itself. 
INDEX

Mots-clés : langue française, féminisation, sociolinguistique, variation, enseignement des langues, histoire de la langue

Keywords : french language, feminization, Sociolinguistics, variation, Language teaching, history of language

\section{AUTEUR}

BÉATRICE FRACCHIOLLA

Université de Paris 8 et Laboratoire SYLED (Paris 3) 